\title{
A risk model for lung cancer screening
}

The publication of several lung cancer screening trials has led to a need to identify who is most likely to benefit from screening. This study sought to validate the Liverpool Lung Project (LLP) risk model in three study populations and to demonstrate its predicted benefit for stratifying patients for CT screening as compared to a screen-all strategy.

The study generated a risk model from risk factor distributions in the LLP Case-Control study data. The performance of this model was assessed by measuring discriminative accuracy, through area under receiver operating characteristic curve. This showed an area under receiver operating characteristic curve of 0.67 to 0.82 in the three study data sets; a greater discrimination than smoking duration. The study also estimated clinical benefit given predicted absolute risk; for a given risk threshold of 5\%, the model achieved a higher proportion of true-positive classifications than a screen-all strategy and strategies based on smoking and family history.

The LLP risk model is convincingly validated to distinguish those likely to develop lung cancer using a predicted 5-year absolute risk. It also shows promise as a selection tool to determine who to screen for lung cancer by unifying important risk factors. Unfortunately, the investigators were unable to include asbestos exposure in the model. The benefits stated are also only predicted; prospective work within a screening trial would validate this.

Overall the LLP risk model shows some promise as a tool to inform selection of patients for lung cancer screening, but further work is needed before it can be used in practice.

- Raji OY, Duffy SW, Abaje OF, et al. Predictive accuracy of the Liverpool Lung Project Risk Model for Stratifying Patients for Computed Tomography Screening for Lung Cancer. Ann Intern Med 2012;157:242-50.

Charles Sharp

Correspondence to Dr Charles Sharp, ST4, Department of Respiratory Medicine, Royal Devon and Exeter NHS Foundation Trust, Barrack Road, Exeter, EX2 5DW, UK; charles.sharp@nhs.net

Competing interests None.

Provenance and peer review Not commissioned; internally peer reviewed.

To cite Sharp C. Thorax 2013;68:893.

Published Online First 27 October 2012

Thorax 2013;68:893. doi:10.1136/thoraxjnl-2012-202846 